\title{
Tablet Inspection and Sorting Machine
}

\author{
Abdur Rehman Riaz \\ Electronic Engineering Department \\ PAF - Karachi Institute of Economics \\ and Technology, Pakistan
}

\author{
M.U. Farooq \\ Electronic Engineering Department \\ PAF - Karachi Institute of Economics \\ and Technology, Pakistan
}

\author{
Areeb Muzaffar Syed \\ Electronic Engineering Department \\ PAF - Karachi Institute of Economics \\ and Technology, Pakistan
}

\begin{abstract}
Tablets are produced in a vast quantity everyday, and which require a proper quality inspection to avoid accidental production issues. The tablet quality checking is mostly done manually which makes it an error-prone process. However, automated solutions for inspection provide much faster and accurate sorting and checking for all tablet shapes, sizes and defects. Such systems require a reliable tablet image analysis mechanism. This paper describes a low cost automated solution for tablet inspection and sorting.
\end{abstract}

\section{Keywords}

Tablet Inspection and Sorting, Machine Vision, LabVIEW, Image Processing

\section{INTRODUCTION}

Pharmaceutical companies all around the world, produce a vast quantity of tablets everyday. This mass production results in various production issues. The issues like wrong dose or extra dose due to difference in weight of tablets etc cause higher operational costs and process downtime. These issues, depending on their level of error, can have very adverse effects and could even prove to be fatal. The various risks associated with the production have created a sense of awareness for improvement of quality and considering a mass production, an artificial intelligent machine based on computer-simulated human act is a must.

Therefore, a reliable machine vision based identification and sorting system is extremely required for reduction in medication errors. An automation to this whole quality assurance process can absolutely add a big value to the production. There are different sorters being used in industry which are designed to handle many shapes and sizes for accurate tablet sorting in order to provide proper assurance of quality.

This paper aims to provide a solution for a low cost machine which can inspect the tablets passing through and check if the tablets match the size and shape of the original tablets. Based on this inspection, automated action will be taken to separate the right tablet from the unwanted ones.

The rest of the paper is arranged as follows. Section 2 describes the design objectives and the related issues. Section 3 provides a literature review of the product. Section 4 provides details on the proposed solution with block diagrams and simu- lations. Finally, Section 5 concludes the paper and discusses the future recommendations.

\section{DESIGN OBJECTIVES, ISSUES \& THEIR ANALYSIS}

\subsection{Design Objectives}

Objectives which are mentioned below are taken care of in the design of this Tablet Inspection and Sorting Machine.

- The Image Analysis techniques are used to find out if quality of images is adequate or not and to detect the presence or absence of material. The techniques analyze the grey level intensities of the pixels of the image and produce measurements and perform different statistical operations.

- The quality of images is improved by using Image Processing and the tools such as Look-Up Tables, Spatial Filters, GreyScale Morphology and Frequency Domain Processing.

- Machine Vision technique is used to check for the missing parts and to check each image and compare it with the original dimensions.

- Vision Acquisition Software is used with three drivers to acquire, save and display pictures.

- Vision Development Module is used for image processing and analysis.

- Vision Assistant Tool of VDA is used to generate a code after the analysis of image is done.

- LabVIEW software is used for most of the work.

- A belt with DC motor is used which will run the tablets and separates them.

\subsection{Is it a suitable technology?}

Currently, the automated inspection and sorting machines are only being used by the high-end laboratories. The smaller laboratories, however, are still using manual inspection methods due to the high costs of automated machines.

This technology of artificial intelligence in pharmaceutical sorting machines is continually growing. In fact, in many countries, companies are bound to have the tablets properly inspected before being able to introduce them in the market. This technology not only allows the pharmaceutical companies to meet the regulations but also opens the door to the international markets because of the proper quality assurance. Therefore, the companies are taking interest in this technology and the sorting industry is improving. 
With this accelerating pace of advancement, the future where these inspection and sorting technologies will have even more potential does not seem far.

\section{LITERATURE REVIEW}

In pharmacy, tablets are a mixture of two or more materials binded together with a binding powder and then molded and pressed into the form of a tablet which is usually circular or disk-shaped. It is very important to monitor different attributes of tablets in the manufacturing process to be able to provide quality assurance.

\subsection{Tablet Analysis}

\subsubsection{Tablet Properties}

- The shapes of tablets could vary but most common shapes are round and oval. The unusual shapes of tablets could cause difficulties in swallowing.

- Tablets are made in such a manner that they are easy to digest but are hard when capped in a bottle.

- Tablets are tested for hardness using hardness tester so that it can be able to resist any kind of stress due to handling, shipping or other factors.

\subsubsection{Advantages of Tablets}

- Tablets can provide a right medicament to diagnose, cure, treat, or prevent diseases.

- Tablets are not only cost effective, if compared with other kinds of medicines but are also convenient in shipping and packaging.

- Their smaller size makes the mass production possible.

- Tablets have many types that are formulated to offer rapid or controlled drug releases i.e. their rates of drug release and durations for the clinical effects could vary as required.

- Tablets are odorless and it is easy to mask the bitter taste of different active substances, making the intake convenient for the patients.

\subsubsection{Disadvantages of Tablets}

- Not suitable in case of unconsciousness and emergency. Also, tablets are not a good choice in case of children and infants.

- Tablets formulation requires a lot of experimentation to find compatibility between the active and inactive substances.

- The granulation technique can add heat or moisture into the substances.

\subsection{Modern Tablet Inspection and Sorting Machines}

3.2.1 Machine types \& manufacturers. There have been many researches made in the field of automated tablet inspection and sorting from researchers all over the world. Following are some models from various pharmaceutical companies.

\section{- Tablet and Capsule Inspection Belt by KEVIN PROCESS} TECHNOLOGIES:

This inspection machine is capable of identifying and removing the defective tablets manually with efficiency and reliability. The tablet may either be accepted or rejected based on the processing, but both are collected in a box. A combination of conveyor belt and rollers ensures that the tablet is checked from all dimensions.

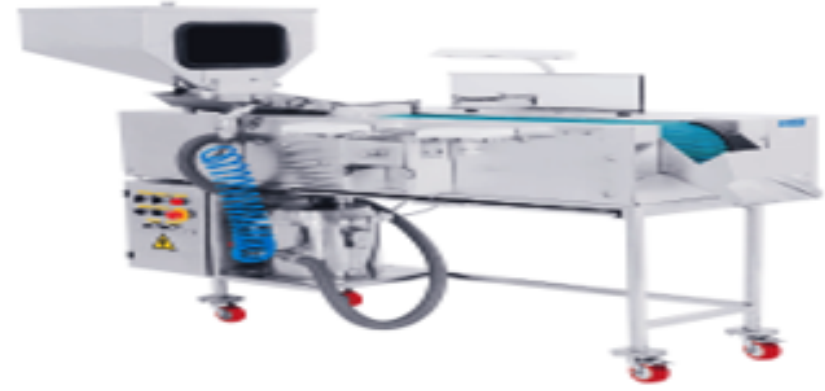

Fig. 1. Tablet and Capsule Inspection Belt

\section{- Tablet Thickness Sorter by KEVIN PROCESS TECHNOLO-} GIES:

This type of machine automatically sorts the plain or coated tablets for thickness. The tablets which do not match the dimensions of the original tablet are rejected. The thickness issues arise in the production process when the layer becomes thick by mistake. Based on the results from thickness identification process, the tablets are automatically separated into 3 different sections depending on their level of thickness.

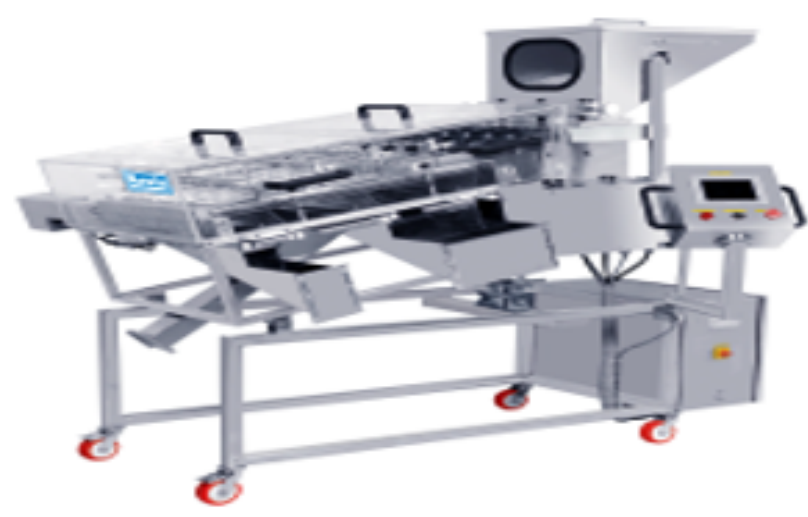

Fig. 2. Tablet Thickness Sorter

-VIM+T: Vision Inspection Machine for Tablets by QUALICAPS:

This machine also checks the tablets from all dimensions using advanced optics methods. It can even check the edges by capturing images from front, back and sides.

-QS: Compact Inspection Sorter Machine by QUALICAPS:

This sorter machine performs inspection process on tablets for many kinds of imperfections like dirt, cracks, imprints and various others. 


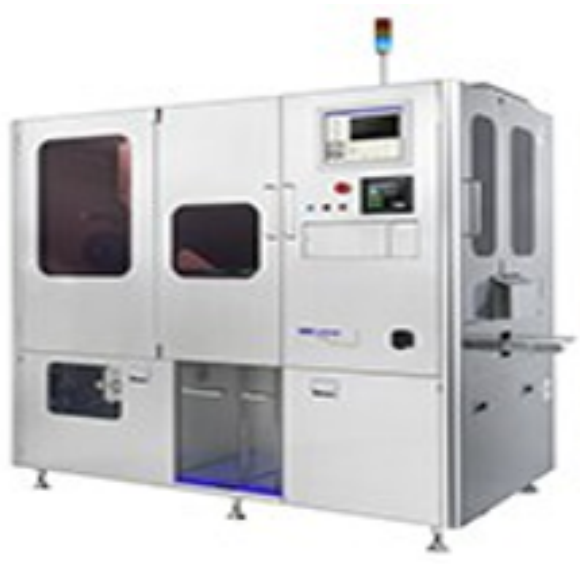

Fig. 3. VIM+T: Vision Inspection Machine

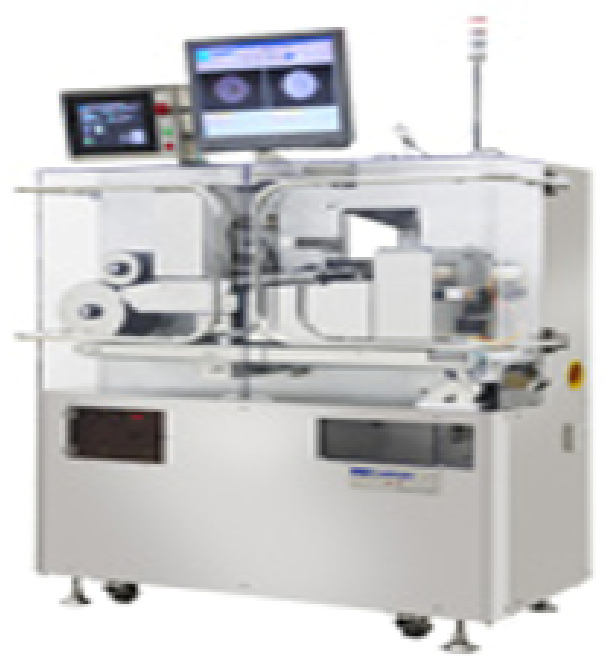

Fig. 4. QS: Compact Inspection Sorter Machine

\subsection{Image Analysis \& Processing Techniques}

To begin with the whole process, a powerful camera to capture the images is used. The captured images act as an input to the software for post-processing to extract the useful information. The final results are used to check if the tablet matches the original dimensions. There are some techniques to perform this analysis and processing, which are as follows:

3.3.1 Image Analysis. It is the extraction of useful information from the input images like finding shapes in an image, removing noise, counting the objects and various other properties of an object using the image processing techniques. Image analysis provides a statistical data of an image based on which it can be determined if the tablet is present or not.

3.3.2 Image Processing. After the image analyzing process, certain image processing techniques are used for further improvement in quality of the image. These techniques can be used to determine certain features that are of interest. Some of the techniques are as follows:
Look-Up Tables:

A Look-up table is used to substitute the new gray scale values for each pixel of the input gray scale image. This technique can be used for improvement in brightness and contrast of certain pixel values with poor brightness and contrast conditions.

\section{Spatial Filters:}

It is a technique to remove noise from the input image, if any. The technique can also be used to smooth and sharpen the image for better results. Certain filters like Gausssian, Laplacian, Sobel filters and many others are used for this technique.

Gray Scale Morphology:

This morphology technique is a transformation which make changes in the input image for specific purposes. The image can be filtered or smoothed and can be altered by making changes in the bright or dark areas.

Frequency Domain Processing:

In this kind of processing, the image is represented in the frequency domain. All the unwanted frequency signals can be removed from the image for further processing.

3.3.3 Machine Vision. Machine Vision technique helps in comparing the input images with the original image to determine if there are some missing parts in the object. The following techniques make use of the machine vision technique for post processing of an image:

\section{Edge Detection:}

It works by checking for the discontinuities in the brightness of an image. Edges of an image can be used to find the projections, width of the object, the intersection points and many other features with an accuracy.

\section{Gauging:}

This technique can be used to measure different features of the object like the length, diameter and others, to compare with the standard image. Based on its result, the comparison process begins and the decision is taken about acceptance and rejection of the object.

3.3.4 Vision Acquisition Software. The NI-IMAQ, NI-IMAQdx, and NI-IMAQ I/O are a set of drivers in LabVIEW which are used to capture, show and save images from different image acquisition devices. Each of the driver package consists of different Virtual Instruments (VIs) which are used for various imaging devices controlling.

3.3.5 Vision Development Module. This module provides image analyzing, processing and machine vision solutions like Edge detection, Optical Character Recognition (OCR) and others, for LabVIEW. It also has a Vision Assistant Tool which prototypes and generates the code by making a series of analysis of the input image.

3.3.6 Optical Sorting. It is an automated process to sort the product based on the attributes like color, shape, size and some structural and chemical compositions, by the help of cameras. This technique is widely used in food processing and pharmaceutical industries because of its accuracy and ability to maximize the production while dealing with all possible errors during production. 


\section{DESIGN SPECIFICATIONS}

\subsection{Overview}

The Tablet Inspection and Sorting Machine consists of a Conveyor Belt with a fixed DC Motor, a Camera to take images of the tablets, some tablets and two boxes for accepted and rejected tablets. The conveyor speed and the alignment sensor of the conveyor belt are controlled to maintain a straight flow of tablets. The camera device captures the images and transfers to the computer for further processing. The results of that post processing checks for the number of tablets, production and rejection rate and decides if any certain tablet is accepted or rejected. The data can also be transmitted wirelessly to the data logging department to keep record.

\subsection{Block Diagrams}

The block diagram of whole system is shown in the following figure. The Image Section captures all the images during the process and applies different image processing techniques. The Conveyor Section moves the belt on which tablet is placed. Finally, the Control Section takes the decision of whether to accept or reject the tablets.

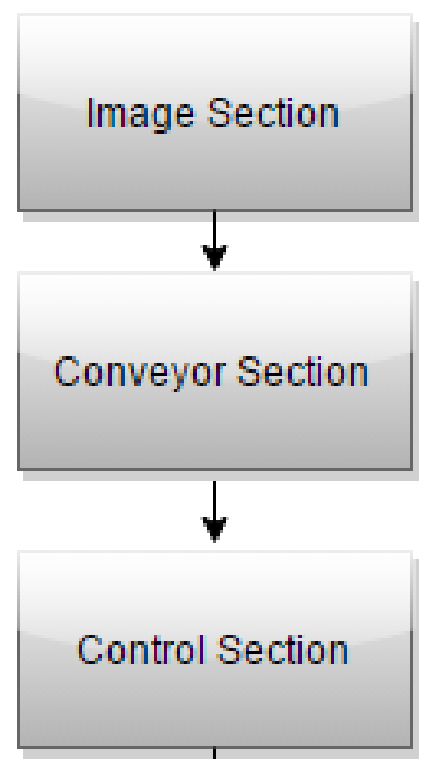

Fig. 5. Block Diagram

\subsection{Labview Working}

4.3.1 Labview Front Panel. The LabVIEW display has three parts as shown in the Fig. 9. The parts are described as follows:

(1) Image Out (It is the live feed from the camera device)

(2) Snap Image (It snaps any particular image frame for recognition)

(3) Template (A part of the captured image is compared with the template image)

Images are continuously taken from the camera device. A pattern is created by cropping any particular region in the image which is then matched with the template.
4.3.2 Labview Block Diagram. There are two loops in the display as shown in the Fig. 9. The loops are described as follows:

Image Acquisition and Operation Loop: This loop is responsible for acquiring the images from camera device. After acquiring the image, it waits for the second loop to generate a trigger and perform the operation of recognition using Image Processing functions. The captured images are first converted into the gray scale which are then sent to the second loop to obtain the template. The image is cropped in any particular region which is sent to this loop for comparison.

User interface loop: This loop is responsible for monitoring the inputs from front panel interface and generating the required trigger action for the first loop to perform matching and taking a decision.

4.3.3 Functions. The block diagram functions are explained in the figure below:

\section{Configures LabVIEW to acquire continuous images from camera connected to PC}

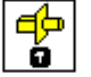

Converts image to grey scale for processing

Saves the template to memory (Learn pattern)

Matches the pattern from live feed

Overlays an indicator to point the desired region

Selects camera

Grabs frames from camera

Fig. 6. Functions

\subsection{Mechanical Hardware}

The mechanical hardware consists of a conveyor belt with a fixed motor to rotate it. The tablets move on the belt while their images are captured with camera during the process. These captured images are analyzed and based on which, the decision about accepting or rejecting the tablet is taken.

\section{CONCLUSION}

\subsection{Conclusion}

With the automation in inspection and sorting, the pharmaceutical companies are able to provide tablets/capsules with proper quality assurance such that they meet the criteria set by the customer. Automatic sorting method may look expensive than the manual sorting method, but it is a onetime expense and provides more accuracy 


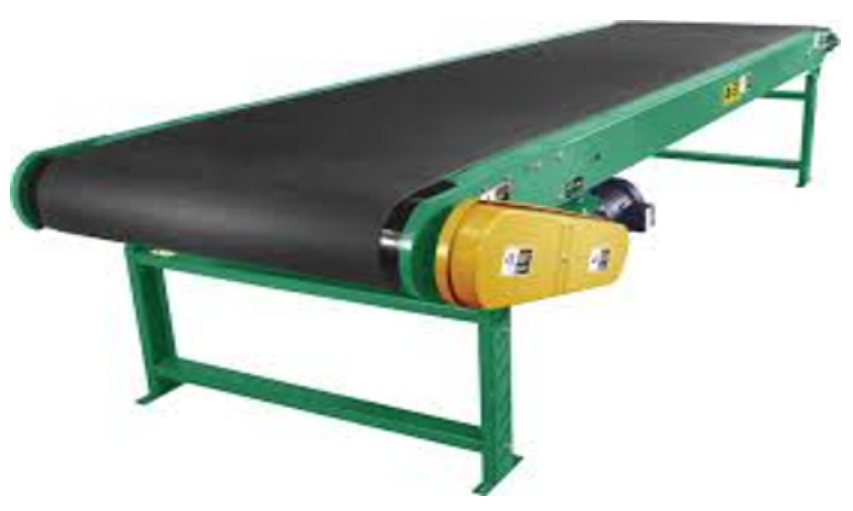

Fig. 7. Mechanical Hardware

than the latter. This paper presented such a low cost automated solution for inspection and sorting. Knowing all the benefits of automation, industries are now making upgrades and are expected to be completely automated within few years.

\subsection{Future Recommendation}

There is still a room for many improvements and modifications in this proposed solution for inspection and sorting of tablets. The project should be expanded with better and huge machinery so it can cater a larger number of tablets in a less time. The software for this machine should be designed by professionals and the necessary equipment including sensors, cameras and other internal mechanisms should be of better quality.

\section{REFERENCES}

[1] 'Products For Tablet Thickness Sorting And Visual Inspection — Pharma Blog'. N.p., 2014. Web. 23 Mar. 2015.

It can be accessed at: www.boschpharma-blog.com

[2] 'Packaging Machinery And Equipment - Packaging Equipment'. N.p., 2015. Web. 23 Mar. 2015.

It can be accessed at: www.busch-machinery.com

[3] Kevin.co.in,. 'Tablet Thickness Sorter'. N.p., 2015. Web. 23 Mar. 2015.

[4] 'Capsule And Tablet Inspection Machines — Qualicaps'. N.p., 2015. Web. 23 Mar. 2015.

It can be accessed at: $w$ ww.qualicaps.com

[5] 'Seidenader Inspection And Sorting Machines For Tablets And Capsules'. 1st ed. Hamburg: KOERBER MEDIPAK, 2013. Web. 23 Mar. 2015.

[6] University of Ljubljana,. 'Automatic Inspection And Sorting Of Tablets \& Capsules'. Ljubljana: Sensum, 2012. Print. SPINE.

[7] Zheng, Hong et al. 'Automatic Sorting Of Chinese Jujube (Zizyphus Jujuba Mill. Cv. Hongxing) Using Chlorophyll Fluorescence And Support Vector Machine'. Journal of Food Engineering 101.4 (2010): 402-408. Web. 23 Mar. 2015. 


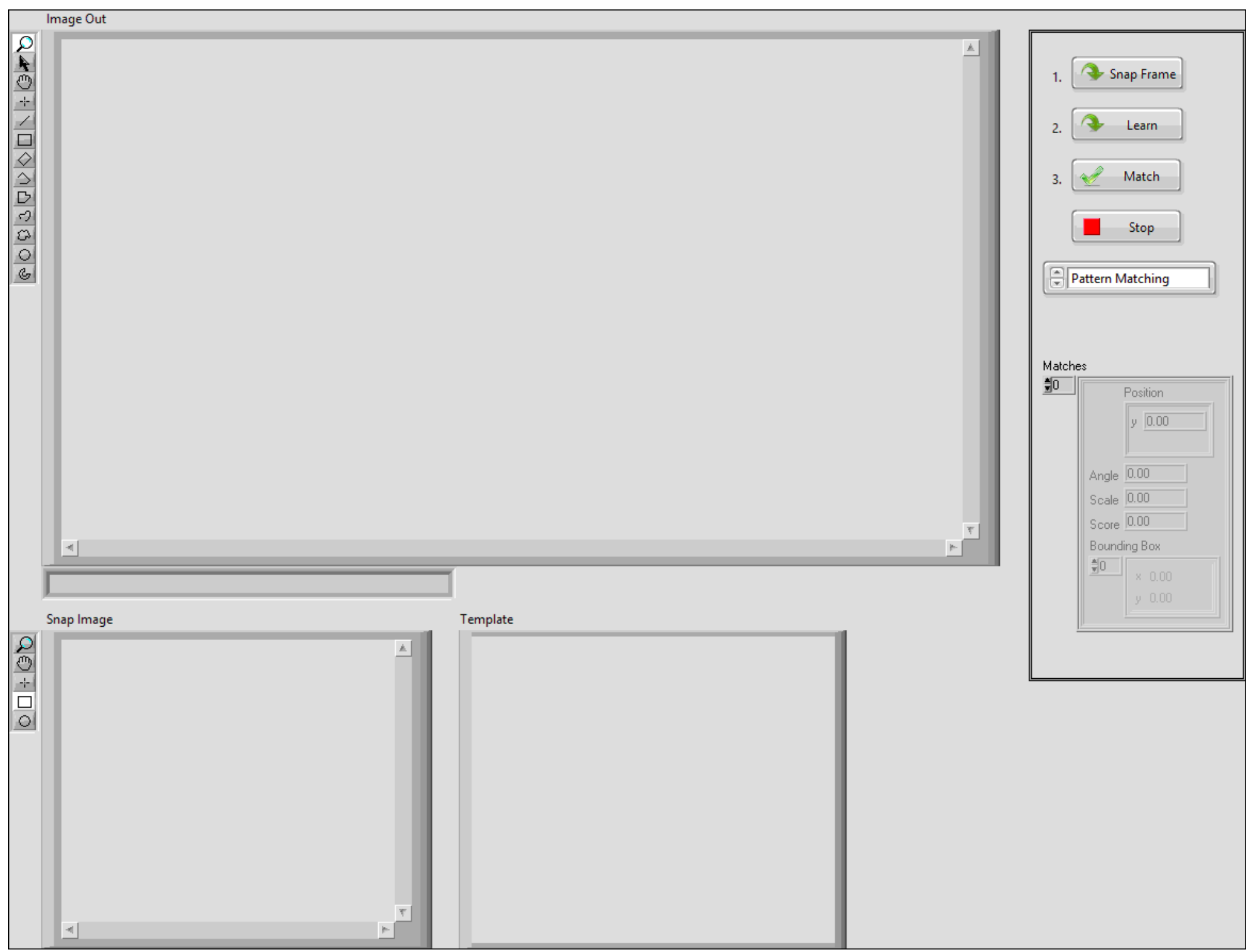

Fig. 8. LabVIEW Display 


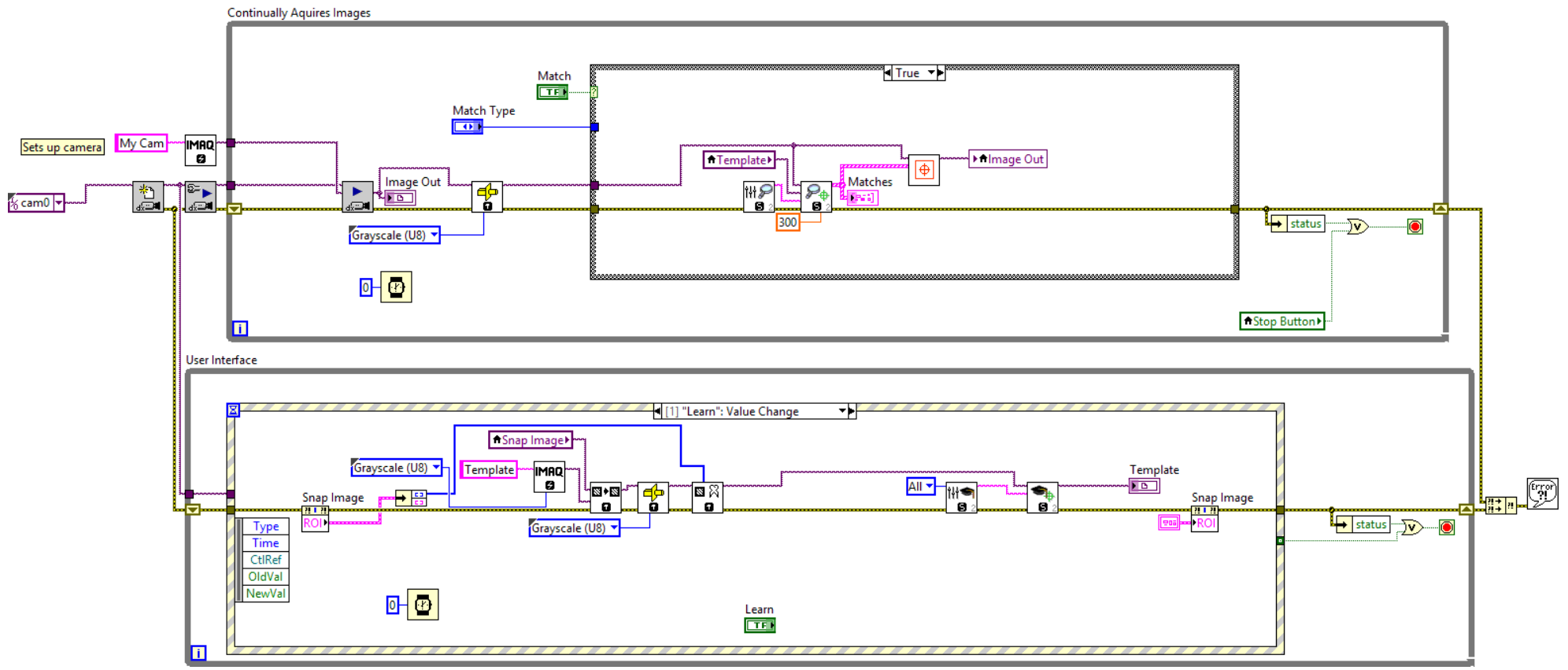

Fig. 9. LabVIEW Block Diagram 